\title{
Results of operative treatment of hypopharyngeal diverticulum
}

\author{
S. B ER TELSEN and A. A A S TED \\ Department of Thoracic Surgery L, Bispebjerg Hospital, and Department of Thoracic Surgery $R$, \\ Rigshospitalet, Copenhagen, Denmark
}

\begin{abstract}
Bertelsen, S. and Aasted, A. (1976). Thorax, 31, 544-547. Results of operative treatment of hypopharyngeal diverticulum. The so-called hypopharyngeal diverticula may be classified, according to their size, localization, and form, into three stages. Small diverticula (first stage) should not be surgically removed, whereas all other diverticula, that is, those causing symptoms, must be surgically removed as soon after diagnosis as possible. Diverticulectomy performed in one stage is the treatment of choice. A series of patients is reviewed. The risk involved in surgery is found to be of minor degree, and the incidence of complications is low. Radiologically demonstrable recurrence may develop in $10 \%$ to $12 \%$ of all patients while clinical symptoms of recurrence are seen in only about $2 \%$.
\end{abstract}

The one-stage operation, that is, extirpation of the diverticulum close to the oesophagus followed by suture of the mucous membrane and hypopharyngeal muscle, has become increasingly common within the last 30 to 40 years, and at present this method must be the treatment of choice (Sweet, 1956; Clagett and Payne, 1960; Kinley, 1966; Holinger and Schild, 1969).

Consideration of the supposed aetiology of the development of hypopharyngeal diverticula has within the last 10-15 years prompted several surgeons to attempt posterior myotomy on the inferior pharyngeal constrictor muscle either as an isolated procedure or combined with extirpation of the diverticulum (Blakeley, Garety, and Smith, 1968; Ellis et al., 1969; Dorsey and Randolph, 1971).

In Scandinavia, Kjærgaard (1941) and Hansen (1950) were the first to publish reports on the favourable results obtained by a one-stage operation; the fact that only minor risks are involved in the procedure has been confirmed in later reviews (Gammelgaard, 1955; Hansen et al., 1973).

The hypopharyngeal diverticulum arises as a protrusion of the pharyngeal mucosa posteriorly between the oblique fibres of the inferior constrictor muscle of the pharynx and the transverse fibres of the cricopharyngeal muscle (Lahey and Warren, 1954).

In the first (initial) stage a bulge of mucosa and submucosa presents through the triangular weak point at the pharyngo-oesophageal junction.
In the second (intermediate) stage the divertio culum is developed. The sac is globular in shaps and dependent. The opening of the diverticuluno into the oesophageal wall is in the vertical plane

In the third (final) stage the diverticulum iQ dragged further and further downward into th $\vec{\delta}$ mediastinum. The opening into the diverticulun is now in the transverse plane, and the true open ing into the oesophagus is converted into a lateras. slit.

\section{PATIENTS}

In the period $1947-71,163$ patients with hypo pharyngeal diverticula were admitted to the thoracic departments of Rigshospitalet and Biso pebjerg Hospitals. The series included $106 \mathrm{men}$ and 57 women; their ages ranged from 34 to 89 with an average of 68 years (Table I).

T A B L E I

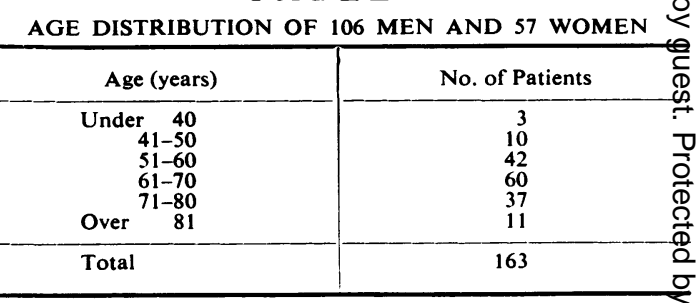


Table II shows the classification into stages of the diverticula in 163 patients (Lahey and Warren, 1954).

T A B L E I I

STAGE DISTRIBUTION

\begin{tabular}{l|c}
\hline Stage & No. of Patients \\
\hline First & 5 \\
Second & 122 \\
Third & 36 \\
\hline
\end{tabular}

A total of 147 patients were submitted to surgery.

Patients with first-stage diverticula were never given surgical treatment.

Of a group of 122 patients with second-stage diverticula, surgery was performed in 112 . In six patients operation was not performed because of severe heart diseases. In one patient operation was not performed because of cerebral apoplexy; one patient died of aspiration pneumonia before operation, and two patients refused treatment.

One of the patients with a third-stage diverticulum died of aspiration pneumonia before surgery could be performed, while operation was performed on the remaining 35 patients in this group.

\section{TECHNIQUE}

Diverticulectomy is performed under general anaesthesia. The diverticulum is exposed through an incision anterior to the lower part of the left sternocleidomastoid muscle. A tube is placed in the oesophagus. After careful dissection of the neck of the diverticulum the latter is extirpated. The mucous membrane and the hypopharyngeal muscles are closed with interrupted silk sutures. Drains must remain in the neck wound for three to four days, and the patient must not have oral fluids for the first 24 hours. Since 1954 the postoperative regimen has included administration of penicillin for six to seven days.

\section{COMPLICATIONS}

Simple wound infection occurred in two patients. Oesophageal fistulae developed in 11 patients; in nine of the latter, the fistulae closed spontaneously within three weeks, while the healing process lasted six weeks in two patients.

Postoperative paresis of the left recurrent laryngeal nerve developed in eight patients; the paresis was of transient nature in five cases but permanent in three.

Cardiac arrhythmia developed in five patients within 48 hours of operation, but digitalization corrected the arrhythmia in all cases. One patient died after an acute myocardial infarction on the fourth day after operation. Clinical symptoms of minor pulmonary embolism were observed in two patients who consequently received anticoagulation therapy. Postoperative pneumonia developed in three patients with third-stage diverticula in whom several episodes of aspiration into the trachea had occurred before operation. One of these patients died.

Only two of 147 surgically treated patients died in hospital after operation.

\section{FOL.LOW-UP}

The patients were followed up for periods of three to 25 years after operation (average 11 years).

SECOND-STAGE DIVERTICULA In the group of 112 surgically treated patients, 36 had died at the time of follow-up. Another group of 23 patients were over 80 years old and they were not personally interviewed, but we were informed by a questionnaire that they were all symptom free.

Clinical and radiological follow-up of 53 patients showed that 47 of these were completely free from symptoms except that radiography disclosed major or minor irregularities at the site of operation in 11 patients.

Symptoms had recurred in three patients, and barium studies disclosed the presence of true recurrences of the diverticula; so far, two of these patients have been subjected to a second operation.

Three patients presented with symptoms of stricture, and barium studies confirmed the presence of a localized stricture at the site of resection of the diverticulum.

Two patients were found to have a 'false' recurrence. Both patients had had two diverticula originally, but only one had been visualized by lateral and postero-anterior radiographs, and hence only one diverticulum had been removed. Both patients were reoperated upon and barium studies after operation were shown to be normal.

THIRD-STAGE DIVERTICULA In the group of 35 surgically treated patients, 14 had died at the time of follow-up; symptoms of recurrence were absent in six patients over the age of 80 years who were not interviewed. Follow-up of the remaining 
15 patients showed that they were free from symptoms except that barium studies disclosed a minor pouch in three but no true recurrence.

Thus, in the series of 97 surgically treated patients who were interviewed, 91 were found to be free from symptoms. However, of 68 patients submitted to radiological examination, changes ranging from minor pouches to a true recurrence of the diverticulum were demonstrable in 14 $(20 \%)$. The incidence of a true recurrence may be $10-12 \%$.

Clinical and radiological examination disclosed a true recurrence in three patients $(2 \%)$.

A stricture at the site of resection of the diverticulum was demonstrable clinically and radiologically in three patients $(2 \%)$; each had been treated by bouginage on several occasions.

Permanent left recurrent nerve paresis occurred in three patients $(2 \%)$.

\section{DISCUSSION}

The age range and the complications after operation in our patients are comparable with those in previously reported series. On the other hand, data on the incidence of recurrence, ranging from 3\% to $12 \%$, are conflicting in some reports.

It cannot be excluded that 'recurrence' in some cases may be due to insufficient dissection on the neck of the diverticulum (Lahey and Warren, 1954).

For instance, in two of our patients it escaped attention at radiological examination and at surgery that two diverticula existed; such double diverticula may be misinterpreted as 'recurrences'. With a view to revealing the presence of more than one diverticulum, barium studies using bilateral projections in the oblique plane in addition to projections in the postero-anterior and lateral planes are routinely recommended.

Radiological examination confirmed recurrence associated with symptoms in $2 \%$ of the patients. Oesophageal changes localized to the site of resection were revealed by radiological examination in 14 of 68 otherwise symptom free patients. These changes may represent surgical sequelae, remnants of a diverticulum which had not been adequately removed, or a genuine recurrence. Radiological examination after operation was not used routinely, and hence the incidence of recurrence cannot reliably be stated. The estimated incidence of genuine recurrence is about $11 \%$.

Although the average age of our patients is high, the mortality after operation is low; it must therefore be emphasized that diverticula causing symptoms should always be excised as soon as possible after diagnosis. Local anaesthesia may be sufficient for the performance of diverticulectomy but general anaesthesia is to be preferred. The risk of severe complications increases with the sizen of the diverticulum.

First-stage diverticula generally should not be surgically removed; they may remain asympto $\vec{\omega}$ matic without increasing in size for years, and they are not easily managed owing to the absence of a well-defined diverticular neck. Thus the risk of surgically induced oesophageal strictures matr be increased.

Because the results obtained by simple extirpa tion of the diverticulum are, in our hands? favourable, it seems hardly relevant to recommend myotomy as a routine; however, for a genuine्ల recurrence reoperation should include combine $\Phi$ myotomy and diverticulectomy. Myotomy was no performed on any patient in the present series.

\section{REFERENCES}

Blakeley, W. R., Garety, E. J., and Smith, D. E (1968). Section of the cricopharyngeus muscle fop dysphagia. Archives of Surgery, 96, 745.

Clagett, O. T. and Payne, W. S. (1960). Surgical trea ment of pulsion diverticula of the hypopharynx? one-stage resection in 478 cases. Diseases of this Chest, 37, 257.

Dorsey, J. M. and Randolph, D. A. (1971). Long-term evaluation of pharyngo-esophageal diverticulecọ tomy. Annals of Surgery, 173, 680.

Ellis, F. H., Jr., Schlegel, J. F., Lynch, V. P., and Payne, W. S. (1969). Cricopharyngeal myotom for pharyngo-esophageal diverticulum. Annals of Surgery, 170, 340.

Gammelgaard, A. (1955). Esophageal diverticula. Re. sults of operative treatment in one stage. Ach Chirurgica Scandinavica, 109, 181.

Hansen, J. B., Jagt, T., Gundtoft, P., and Sørense $\frac{\bigcirc}{P}$, H. R. (1973). Pharyngo-oesophageal diverticular A clinical and cineradiographic follow-up stu\&. of 23 cases treated by diverticulectomy. Scan dinavian Journal of Thoracic and Cardiovasculam Surgery, 7, 81.

Hansen, J. L. (1950). Oesophagusdiverticler. Ugeskrin for Loger, 112, 710.

Holinger, P. H. and Schild, J. A. (1969). The Zenkers (hypopharyngeal) diverticulum. Annals of Ot尺 logy, Rhinology and Laryngology, 78, 679.

Kinley, C. E. (1966). The management of hypopharyngeal diverticulum. Canadian Journal of Surger: 2, 27.

Kjærgaard, S. (1941). Om operativ behandling 争 oesophagusdivertikler. Ugeskrift for Lager, $10 \%$. 989. 
Lahey, F. H. and Warren, K. W. (1954). Esophageal diverticula. Surgery, Gynecology and Obstetrics, 98, 1.

Sweet, R. H. (1956). Excision of diverticulum of the pharyngo-esophageal junction and lower esopha- gus by means of the one stage procedure. Annals of Surgery, 143, 433.

Requests for reprints to: Dr. S. Bertelsen, Solbakkevaj 24, 2820 Gentofte, Denmark. 